
allemande

48-1 | 2016

Les expériences coloniales allemandes : échanges, transferts, circulations

\title{
Die Aneignungsungleichheiten in Hinsicht auf die regionale Energieerzeugung im Zeitalter der ökologischen Wende
}

Soziologische Analyse eines kooperativen Instrumentes im Bereich „Bürgerwindkraft“ im Elsass

Philippe Hamman

\section{OpenEdition \\ Journals}

Édition électronique

URL : https://journals.openedition.org/allemagne/381

DOI : 10.4000/allemagne.381

ISSN : 2605-7913

Éditeur

Société d'études allemandes

Édition imprimée

Date de publication : 15 juin 2016

Pagination : 193-214

ISSN : 0035-0974

Référence électronique

Philippe Hamman, „Die Aneignungsungleichheiten in Hinsicht auf die regionale Energieerzeugung im Zeitalter der ökologischen Wende", Revue d'Allemagne et des pays de langue allemande [Online], 48-1 I 2016, Online erschienen am: 13 Dezember 2017, abgerufen am 22 Mai 2021. URL: http:// journals.openedition.org/allemagne/381 ; DOI: https://doi.org/10.4000/allemagne.381 


\section{Die Aneignungsungleichheiten in Hinsicht auf die regionale Energieerzeugung im Zeitalter der ökologischen Wende} Soziologische Analyse eines kooperativen Instrumentes im Bereich „Bürgerwindkraft“ im Elsass

\section{- Philippe Hamman*}

\section{Einleitung}

2012 wurde im Rahmen der zweiten Rio-Konferenz die progressive Ablösung der fossilen Energieträger durch erneuerbare Energien beschlossen. Somit wurde die Energiewende zum Motor der „Green Economy“ “(1). Angesichts der Risiken des Klimawandels haben sich die Mitgliedstaaten der EU dazu entschlossen, ihren CO2-Ausstoß bis 2050 im Vergleich zu 1990 um 80\% zu reduzieren. In der Folge hat sich Frankreich verpflichtet, bis 2020 bei der Stromerzeugung einen Anteil von 23\% aus erneuerbaren Energiequellen zu beziehen. Diese konkreten Zahlen und Vorgaben bilden den Rahmen der europäischen Energiewende und zielen auf das Erreichen einer sogenannten nachhaltigen Entwicklung ab. Die „Green Economy“ scheint das Ziel zu sein, das es zu erreichen gilt, in einer Welt, die zunehmend mit den Grenzen des Wachstums und der Ressourcen konfrontiert ist. Statt das herrschende wirtschaftliche Bezugssystem infrage zu stellen, wird auf „grünes Wachstum“ gesetzt, und der technologische Fortschritt soll grünen und weniger energieintensiven Lebensweisen Vorschub leisten ${ }^{(2)}$.

* Professor für Soziologie am Institut für Stadtsoziologie und Raumplanung der Fakultät für Sozialwissenschaften, Universität Straßburg: <phamman@unistra.fr>.

1 Ian Bailey, Federico Caprotti, „The Green Economy: Functional Domains and Theoretical Directions of Enquiry“, Environment and Planning A, 46/8 (2014), S. 1797-1813; Stewart LockIE, David A. Sonnenfeld, Dana R. Fisher (Hg.), The Routledge International Handbook of Social and Environmental Change, London/New York, Routledge, 2014, Abschnitt II.

2 Hamil Pearsall, Joseph Pierce, Robert Krueger, „Whither Rio+20?: Demanding a Politics and Practice of Socially Just Sustainability“, Local Environment, $17 / 9$ (2012), S. 935; Guillaume CHRISten, Philippe Hamman, Mathias Jehling, Maurice Wintz (Hg.), Erneuerbare Energien-Systeme in Deutschland und Frankreich / Systèmes énergétiques renouvelables en France et en Allemagne, Paris, Orizons, 2014. 
Die konkrete Realisierung eines Bezugsystems der nachhaltigen Entwicklung setzt zunächst eine „lokale“ Verortung der öffentlichen oder privaten Initiativen voraus ${ }^{(3)}$. Was die Energieerzeugung angeht, ist dies gleich aus mehreren Gründen unerlässlich: Für die Mobilisierung der verfügbaren lokalen Ressourcen, das Miteinbeziehen der lokalen Möglichkeiten, der örtlichen bodenklimatischen Gegebenheiten, von Wind und Sonneneinstrahlung. Aber auch aus wirtschaftlichen Gründen: als Nische innerhalb der "Green Economy“ unterliegen auch die erneuerbaren Energien den Gesetzen des Marktes ${ }^{(4)}$. Auch auf soziologischer Ebene stellt die Energiewende eine Herausforderung dar, besonders, was die von der Politik gewünschte, breite Partizipation aller Bevölkerungsschichten betrifft ${ }^{(5)}$. Partizipation ist bei öffentlichen Umweltprojekten inzwischen zur Norm geworden ${ }^{(6)}$; eine wichtige Rolle spielen dabei die Sensibilisierung und Einbeziehung der verschiedenen Akteure, besonders in Bezug auf den Einsatz neuer, sogenannter „ökologischer" Technologien, wie es zum Beispiel bei der Windkraft der Fall ist ${ }^{(7)}$. Dies ist ein Hinweis auf die gewachsene Rolle von Prozessen und Verfahrensweisen in der Nachhaltigkeitspolitik, wobei die eigentlichen Inhalte zuweilen leider in den Hintergrund treten ${ }^{(8)}$. Dabei ist Partizipation im Umweltschutz keine Selbstverständlichkeit. Es wird oft über den Graben hinweggesehen, der beispielsweise das urbane Marketing um Öko-Stadtviertel von dem tatsächlichen, tagtäglichen Erleben der Bevölkerung trennt ${ }^{(9)}$. Oft wir in der Umweltpolitik der soziale Aspekt vernachlässigt ${ }^{(10)}$, besonders beim Thema der umweltbezogenen sozialen Ungleichheiten, welches dieser Artikel untersucht.

Energie ist mehr als eine bloße, technische Variable; es geht um gesellschaftspolitische Entscheidungen, an denen Institutionen und politische, wirtschaftliche und soziale Systeme beteiligt sind. Energieerzeugung und -verteilung beruhen auf einer Vielfalt von interagierenden Akteuren: Entscheider, Kollektivitäten, Verbraucher...

3 Philippe Hamman, „Urban Sustainable Development and the Challenge of French Metropolitan Strategies“, Urban Research and Practice, 2/2 (2009), S. 138-157; Philippe Hamman, Sociologie urbaine et développement durable, Brussels, De Boeck, 2012.

4 Bailey/ Caprotti, „The Green Economy“ (Anm. 1).

5 Marit Rosol, „Public Participation in Post-Fordist Urban Green Space Governance: The Case of Community Gardens in Berlin“, International Journal of Urban and Regional Research, 34/3 (2010), S. 548563; Meg Holden, „Public Participation and Local Sustainability: Questioning a Common Agenda in Urban Governance“, International Journal of Urban and Regional Research, 35/2 (2011), S. 312-329.

6 Jason Chilvers, „Deliberative and Participatory Approaches in Environmental Geography“, in: Noel Castree, David Demeritt, Diana Liverman, Bruce Rhoads (Hg.), A Companion to Environmental Geography, Hoboken, Wiley-Blackwell, 2009, S. 400-417.

7 David Toкe, „Ecological Modernisation, Social Movements and Renewable Energy“, Environmental Politics, 20/1 (2011), S. 60-77.

8 Giles Atrinson, Simon Dietz, Eric Neumayer (Hg.), Handbook of Sustainable Development, Cheltenham, Edward Elgar Publishing, 2008, Abschnitt V; Philippe Hamman, „La gouvernance de la ,ville durable ou les enjeux d’une perspective procédurale“, in: Isabelle Hajeк, Philippe Hamman (Hg.), $L a$ gouvernance de la ville durable entre déclin et réinventions, Rennes, Presses universitaires de Rennes, 2015, S. 33-61.

9 Zum Beispiel bei der Sonnenenergie: Tim Freytag, Stefan Gössling, Samuel Mössner, „Living the Green City: Freiburg's Solarsiedlung between Narratives and Practices of Sustainable Urban Development", Local Environment, 19/6 (2014), S. 644-659.

10 Atkinson/ Dietz/ Neumayer (Hg.), Handbook of Sustainable Development (Anm. 8), Abschnitt III. 
Die Politik zur Einführung der erneuerbaren Energien unterscheidet sich teils deutlich von einem Land zum andern - in Europa wird dies besonders am Gegensatz zwischen Deutschland und Frankreich deutlich: zwei Länder, deren geschichtlicher und politischer Kontext ebenso unterschiedlich ist, wie ihre Energiemärkte ${ }^{(11)}$.

Die Nutzung und die Verbreitung einer Energiequelle gleicht einem soziotechnischen System, das auf drei interagierenden Säulen gründet: Die Energiequelle, die technischen Mittel, dank derer die Ressource nutzbar gemacht wird (beispielsweise Wind- und Sonnenenergie) und das technisch-wirtschaftliche Modell ${ }^{(12)}$. Letzteres bezeichnet ein Kollektiv von Akteuren, welche die Energietechnik entwickeln und vertreiben und die verschiedenen Ausrichtungen, die ihnen vorgegeben werden, in bestimmten Bahnen lenken. Diese Ausrichtungen können die unterschiedlichsten Formen annehmen, von der „Wachstumsrücknahme“ bis zum „grünen Wachstum“. In der soziologischen Literatur wurde bewiesen, dass die erneuerbaren Energien hauptsächlich über den Markt in die Gesellschaft Einzug halten - mit dem Ziel, einen Industriezweig weiterzuentwickeln ${ }^{(13)}$. Dies beruht vor allem auf technischer Innovation, die als linear wahrgenommen wird (Erfindung, Demonstration, Verbreitung) und auf dem Zusammenspiel von Marktmechanismen und Innovation - Es geht darum, mithilfe der „Green Technology“ neue Gewinnquellen zu erschließen ${ }^{(14)}$. Parallel zu dieser industriellen Bewegung existieren auch kollektive Initiativen, die von ihren Trägerorganisationen und den Gemeinden, die sie aufgreifen, als „Bürgerbewegungen“ dargestellt werden. Sie nehmen oft die Form von Energiegenossenschaften an und streben eine Dezentralisierung der Energieproduktion an, mit dem Ziel, die einzelnen Regionen zu unabhängigen Energieproduzenten auszubauen. Es geht ihnen um größere Unabhängigkeit durch den progressiven Austritt aus der industriellen Logik. Am weitesten verbreitet sind sie in Nordeuropa, z. B. in Deutschland, wo es 2012 bereits über 750 Energiegenossenschaften gab $^{(15)}$. Die Umsetzung der Energiewende in Deutschland beruht auf lokalen und regionalen Initiativen, sowohl in den Städten, als auch auf dem Land, die auf lokalen genossenschaftlichen Besitz- und Organisationsstrukturen aufbauen und eine Neudefinition des Konzepts

11 Aurélien Évrard, „Political Parties and Policy Change: Explaining the Impact of French and German Greens on Energy Policy“, Journal of Comparative Policy Analysis, 14/4 (2012), S. 275-291; CHRISTEN/ Hamman/ Jehling/ Wintz (Hg.), Erneuerbare Energien-Systeme in Deutschland und Frankreich (Anm. 2).

12 Laure Dobigny, „Changement énergétique et rapport au monde“, in: Marie-Jo Menozzi, Fabrice Flipo, Dominique PÉcaud (Hg.), Énergie et société. Sciences, gouvernances et usages, Aix-en-Provence, Édisud, 2009, S. 201-210.

13 Christen/ Hamman/ Jehling/ Wintz (Hg.), Erneuerbare Energien-Systeme in Deutschland und Frankreich (Anm. 2); Guillaume Christen, Philippe Hamman, Transition énergétique et inégalités environnementales: énergies renouvelables et implications citoyennes en Alsace, Strasbourg, Presses universitaires de Strasbourg, 2015.

14 Rikke Lyвæк, Thomas Budde Christensen, Tyge KJÆr, „Governing Innovation for Sustainable Development in the Danish Biogas Sector - a Historical Overview and Analysis of Innovation", Sustainable Development, 21/3 (2013), S. 171-182.

15 Noémie Poize, Andreas RüDINGER, „Projets citoyens pour la production d'énergie renouvelable: une comparaison France-Allemagne“, Working papers IDDRI, 1 (2014), URL: http://www.iddri.org/Publications/Collections/Idees-pour-le-debat/WP0114_NP\%20AR_projets\%20citoyens.pdf. 
des „Gemeinguts“ erfordern ${ }^{(16)}$. In Frankreich ist das Phänomen der Energiegenossenschaften jünger - es gibt sie erst seit ca. zehn Jahren - und im Umfang begrenzter: Ein erstes Windkraftprojekt mit Bürgerfinanzierung, Éoliennes au Pays de Vilaine (Windräder im Pays de Vilaine), wurde 2003 in der Region Ille-et-Vilaine begonnen und am 14. Juni 2014 in Betrieb genommen. Zieht man die lokalen Erfahrungen in Betracht, existiert noch ein dritter Weg, jenseits dieser Dualität: ein Mittelweg zwischen diesen beiden Vorgehensweisen, wie er in den Regionen existiert. Dies zeigt auch eine gewisse Widersprüchlichkeit bei den lokalen Initiativen auf: zwischen der Schaffung einer eigenen, dezentralisierten Infrastruktur und der lokalen Vor-OrtDemonstration eines globalen, neoliberalen Bezugssystems ${ }^{(17)}$.

Verdeutlichen lässt sich dies anhand eines konkreten Beispiels: die Errichtung eines Windparks mit zehn Windrädern Nahe einer französischen Ortschaft, S., im Elsass. Der Träger ist ein französischer Energieerzeuger, der sich auf die Erzeugung und den Vertrieb von Strom aus erneuerbaren Energien spezialisiert hat: EDF Énergies Nouvelles, eine Tochtergesellschaft von Électricité de France (EDF). Das Besondere an diesem Fall ist die Hybridisierung der beiden beschriebenen Entwicklungsmodelle: Von den zehn Windrädern werden zwei von einem Bürgerbeteiligungsmodell finanziert und verwaltet. Diese partielle genossenschaftliche Dimension dieses Windparks ist bemerkenswert, insbesondere die Modalitäten und die Instrumente zur Einbindung der Bevölkerung, da die Windenergie zweifellos diejenige unter den erneuerbaren Energien ist, die am meisten Streitpotenzial bezüglich negativer Auswirkungen auf die Umwelt und die Gesundheit der Anwohner mit sich bringt ${ }^{(18)}$.

Wir wollen, um es deutlicher auszudrücken, zwei sich ergänzende Aspekte festhalten. Einerseits ist es erwiesen, dass das Ziel eines Bürgerbeteiligungsdispositivs nicht nur die Einbindung der Nutzer in die Energiewende ist, sondern auch, neue Governance-Modelle zu etablieren, die eine soziale Aneignung der Energiefrage durch die Bevölkerung ermöglichen ${ }^{(19)}$. Hier blicken wir durch das Prisma der Partizipation auf die Beziehungen zwischen Sozialem und Ökologie: Es besteht eine klassische Verbindung, welche dem weitverbreiteten Glauben entspricht, dass der beste Weg zur Nachhaltigkeit die Einbindung der Bürger ist ${ }^{(20)}$. Doch andererseits stößt sich die Beziehung zwischen Partizipation und Umwelt an Ungleichheiten: Die verschiedenen Akteure haben unterschiedliche Lebensumstände, verschiedene Fähigkeiten und Sichtweisen, insbesondere was Wissen, Kompetenzen und Macht betrifft. So wird die Partizipation

16 Timothy Moss, Sören Becker, Matthias Naumann, „Whose Energy Transition is it, anyway? Organisation and Ownership of the Energiewende in Villages, Cities and Regions“, Local Environment, online first: 19.05.2014, DOI: 10.1080/13549839.2014.915799.

17 Alain Nadaï, Olivier Labussière, Ariane Debourdeau, Yannick Régnier, Laure Dobigny, „French Policy Localism: Surfing on ,Positive Energie Territories' (Tepos)“, Energy Policy, 78 (2015), S. 281-291.

18 Jøran SolLI, „Where the Eagles Dare? Enacting Resistance to Wind Farms through Hybrid Collectives“, Environmental Politics, 19/1 (2010), S. 45-60.

19 Laure Dobigny, „Produire et échanger localement son énergie: dynamiques et solidarités à l'œuvre dans les communes rurales“, in: Christian Ferault, Nicole Mathieu, François Papy (Hg.), Nouveaux rapports à la nature dans les campagnes, Versailles, Quae, 2012, S. 139-152.

20 Zum Beispiel, Ton Dassen, Eva Kunseler, Lieke Michiels van Kessenich, „The Sustainable City: An Analytical-Deliberative Approach to Assess Policy in the Context of Sustainable Urban Development", Sustainable Development, 21/3 (2013), S. 193-205. 
nicht nur zum Spiegel der sozialen Ungleichheit, sie institutionalisiert sie auch ${ }^{(21)}$ und stört damit die soziale Identität der Akteure ${ }^{(22)}$. In diesem Artikel betrachten wir die umweltbezogenen sozialen Ungleichheiten anhand der partizipativen Instrumente.

Der Artikel ist in vier Teile gegliedert. Im ersten wird der Rahmen festgelegt: Es werden die nationalen Energiepolitiken Frankreichs und Deutschlands verglichen und der regionale Kontext der Energiewende im Elsass erörtert, sowie das partizipative Bürgerbeteiligungsmodell, welches in S. umgesetzt wurde, und schließlich die angewandte Methodik der Studie. Der zweite Teil befasst sich detaillierter mit der erarbeiteten soziologischen Problematik, um dieses Modell in Bezug auf die ökologischen Ungleichheiten hin zu untersuchen. Anschließend befassen wir uns mit den empirischen Ergebnissen aus dem Konzept der Bürgerbeteiligung (3. Teil) und seine Akzeptanz in der Bevölkerung (4. Teil): Wer sind die Träger und welches Wissen setzen sie Voraus, mit welchen Konsequenzen für die Aneignungsmöglichkeiten durch die lokale Bevölkerung? Dazu untersuchen wir das Phänomen der Professionalisierung von selbsternannten „Bürgerinitiativen“ im weiteren dynamischen Kontext der Vereinnahmung von Umweltprojekten durch spezialisierte und finanzstarke Akteure.

\section{Kontext, Erläuterung des Incentive-Instrumentes und Methodik}

Vergleichende Analyse der Energiepolitik in Frankreich und Deutschland im Hinblick auf die erneuerbaren Energien

Frankreich und Deutschland verfügen über unterschiedliche Energiesysteme; die Energiewende muss dieser mittelfristig fortbestehenden Tatsache Rechnung tragen.

In Deutschland stand die Förderung der erneuerbaren Energien bereits Ende der 80er Jahre auf der politischen Agenda ${ }^{(23)}$. Die deutsche Kernkraftförderung war seit Mitte der 70er Jahre umstritten, und die deutsche Anti-Atomkraft-Bewegung hat sich Anfang der 80er Jahre mit dem politischen Aufstieg der Grünen sowie der Nuklearkatastrophe von Tschernobyl im April 1986 noch verstärkt ${ }^{(24)}$. Anders als in Frankreich erschienen die erneuerbaren Energien deswegen als Alternative zur allesbeherrschenden Kernkraft. Einen wichtigen Beitrag zum Ausbau der erneuerbaren Energien leistete das Stromeinsparungsgesetz von 1991, das einen zwanzig Jahre gültigen Festpreis für den Aufkauf von Solarstrom garantierte ${ }^{(25)}$. 1998 setzte sich die Regierung Schröder (Koalitionsregierung zwischen SPD und Grünen) den Atomausstieg zum Ziel ${ }^{(26)} .2004$ garantierte ein neues Gesetz noch vorteilhaftere Preise für den Verkauf von Solar-

21 Amy Douglas, „The Politics of Environmental Mediation“, Ecology Law Quarterly, 11/1 (1983), S. 1-19.

22 Jeffrey B. JACQUET, Richard C. STEDMAN, ,The Risk of Social-psychological Disruption as an Impact of Energy Development and Environmental Change“, Journal of Environmental Planning and Management, 57/9 (2014), S. 1285-1304.

23 Volkmar Lauber, Lutz Mez, „Three Decades on Renewable Electricity Policies in Germany“, Energy and Environment, 15/4 (2004), S. 599-623.

24 Gilles Leroux, „Les Allemands et le nucléaire: histoire d'un double défi“, Revue d'Allemagne et des Pays de langue allemande, 45/1 (2013), S. 54-56.

25 Staffan Jacobsson, Volkmar Lauber, „The Politics and Policy of Energy System Transformation. Explaining the German Diffusion of Renewable Energy Technology“, Energy Policy, 34/3 (2006), S. 256-276.

26 A. Évrard, „Political Parties and Policy Change“ (Anm. 11). 
strom. Dabei wurde die Preisberechnung degressiv angelegt, um einen Anreiz für technische Innovation zu setzen. Die Effekte dieser öffentlichen Anreizpolitik werden zusätzlich dadurch verstärkt, dass sich Unternehmen entwickeln, die einen eigenen, neuen Industriezweig schaffen, dessen Bandbreite von der Entwicklung und Herstellung der nötigen Materialien bis hin zu Anlagenbau und Energienutzung reicht ${ }^{(27)}$. So erzeugte Deutschland im Jahr 2009 ca. fünf Mal mehr Energie aus Windkraft als Frankreich, sieben Mal mehr Energie aus Biomasse und 37 Mal mehr Solarenergie ${ }^{(28)}$.

Die Nuklearkatastrophe von Fukushima hat die Wende in der deutschen Energiepolitik noch beschleunigt. Im Sommer 2011 beginnt die Bundesregierung mit dem „Zweiten Atomausstieg“: Eine Reihe von Gesetzen und Anreizen sollen den Ausbau der erneuerbaren Energien weiter fördern. Das erste dieser Gesetze, das zum ersten Januar 2012 in Kraft getreten ist, hat zum Ziel, bis 2050 80\% des deutschen Strombedarfs aus erneuerbaren Energien zu decken. Dabei werden die Sektoren Geothermie, Offshore-Windparks und Biogasanlagen gefördert. Außerdem enthält das Gesetz vom 6. Juni 2011 verschiedene Bestimmungen zur Förderung und zum Ausbau der erneuerbaren Energien im urbanen Raum sowie zur Vereinfachung der Errichtung von Solar- und Windkraftparks.

In Frankreich wurde in den 70er Jahren angesichts der Öl-Krise und mit dem Argument der Energieunabhängigkeit ein ganzer Industriezweig rund um die Kernkraft aufgebaut. Die erneuerbaren Energien hatten folglich lange Zeit keine Priorität. Erst 1999 wurden erste Fördermaßnahmen für Solarenergie beschlossen und eine Abnahmegarantie mit Mindestpreis für Solarstrom angeboten. Der garantierte Ankauf von Strom aus erneuerbaren Energien durch EDF wurde erst im Jahr 2000 gesetzlich verankert, also viel später als in Deutschland. Die Anreize waren von Anfang an geringer als in Deutschland; außerdem werden seit 2010 die garantierten Ankaufspreise nach und nach gesenkt ${ }^{(29)}$. Zudem fokussierten sich die französischen Unternehmen vornehmlich auf Produktion und Nutzung der Energien ${ }^{(30)}$, so dass ein großer Teil der nötigen Materialien importiert werden muss.

Dennoch darf nicht voreilig auf einen kompletten Gegensatz zwischen deutschem und französischem Modell geschlossen werden. Es wird oft der sogenannte norddeutsche „Bürgerwindpark“ angeführt, also regionale, lokale Initiativen und Genossenschaftsparks, bei denen auch die Umstrukturierungsdynamiken der Industriehäfen, die starke Präsenz der Bauern und Bezüge zu fest in der Gesellschaft verankerten kollektiven Strukturen (Vereinswesen) eine Rolle spielen. Dieses Modell habe die anarchische Vermehrung der Windräder (die sogenannte „Verspargelung“) verhindert und es ermöglicht, dass die Gewinne der Windparks in den Gemeinden verbleiben ${ }^{(31)}$.

27 Ariane DebourdeAu, „De la ,solution` au ,problème‘. Les problématisations de l'obligation d'achat de l'énergie solaire photovoltaïque en France et en Allemagne“, Politix, 95 (2011), S. 103-127.

28 IDDRI, „L'énergie en Allemagne et en France, une comparaison instructive“, Les Cahiers de Global Chance, 30 (2011), URL: http://www.global-chance.org/L-energie-en-Allemagne-et-en-France-unecomparaison-instructive.

29 Ebd.

30 A. Debourdeau, „De la ,solution“ au ,problème““ (Anm. 27).

31 Werner KRAuss, „The ,Dingpolitik“ of Wind Energy in Northern German Landscapes: an Ethnographic Case Study“, Landscape Research, 35/2 (2010), S. 198-208. 
Doch dies darf nicht den Blick auf die Offshore-Projekte verstellen, die für ein globales industrielles und finanzielles Modell stehen, welches nicht von lokalen, sondern von nationalen Interessen bestimmt ist. Im Grunde genommen machen die Unterschiede zwischen den beiden Ländern und die geteilte politische Dimension des Unterfangens eine lokale Herangehensweise unverzichtbar, was eine Untersuchung am Beispiel des Elsass umso aussagekräftiger macht.

\section{Der regionale Kontext der Energiewende im Elsass}

Die Entwicklung von Anreizen zur Beteiligung der Bevölkerung an der Energiewende anhand eines konkreten, elsässischen Fallbeispiels ist umso lehrreicher, als diese Region an der deutsch-französischen Grenze liegt, also „mittendrin“, zwischen den beiden energiepolitischen Herangehensweisen. Der Rhein ist heute zum Symbol eines deutsch-französischen Kooperationsgrenzgebietes geworden ${ }^{(32)}$. Seine Nutzung als Energiequelle konkretisiert die Natur als grenzüberschreitendes, verbindendes Element. So hat zum Beispiel der deutsch-französische Vertrag vom 4. Juli 1969 über den Ausbau des Rheins die Errichtung zweier deutsch-französischer Wasserkraftwerke in Gambsheim/Freistett und Iffezheim/Beinheim ermöglicht. Der Bau des Kernkraftwerks Fessenheim, von 1971 bis 1978, ist ein weiteres Beispiel für diese Zusammenarbeit; es wurde von drei Energieunternehmen finanziert: der französischen EDF (67,5\%), der deutschen EnBW ${ }^{(33)}(17,5 \%)$ und einem Schweizer Konsortium (NOK, EOS und $\left.\mathrm{BKW}^{(34)}\right)(15 \%)$.

Fessenheim steht auch für die erste Demonstration der französischen Anti-Atomkraft-Bewegung: Am 12. April 1971 nahmen über 1500 Menschen an einem vom Comité pour la Sauvegarde de Fessenheim et de la plaine du Rhin (Komitee zur Rettung von Fessenheim und dem Rheintal) organisierten Protestmarsch teil. Diese Protestkundgebung hat zu einer nachhaltigen Bewusstseinswerdung geführt und die zunehmende Wichtigkeit der Rolle der Umweltschutzorganisationen bei der Erschließung des Elsass' verdeutlicht, die zu unverzichtbaren Ansprechpartnern der öffentlichen Diskussion in der Region geworden sind ${ }^{(35)}$. Die ökologischen Werte dieses dichten und technisch kompetenten Netzwerkes haben das Potenzial, den Ausbau alternativer Energien und Konzepte zu fördern.

Doch der Rhein versinnbildlicht auch die Trennung zwischen unterschiedlichen nationalen Antworten auf die energetischen und klimatischen Herausforderungen. Während in Deutschland nach der Katastrophe von Fukushima beschlossen wurde, erst die acht ältesten Reaktoren des Landes, die für $40 \%$ der Stromerzeugung sorgten, abzuschalten, und bis 2022 in einem zweiten Schritt auch die letzten neun vom Netz zu nehmen, setzt man in Frankreich bei der Energiewende auf einen Mix zwischen Kernkraft und den Ausbau erneuerbarer Energien. Das Elsass als Grenzregion liegt zwischen diesen beiden

32 Philippe Hamman, Sociologie des espaces-frontières. Les relations transfrontalières autour des frontières françaises de l'Est, Strasbourg, Presses universitaires de Strasbourg, 2013.

33 Die Energie Baden-Württemberg AG ist der drittgrößte Energieanbieter Deutschlands. 2005 wurde EDF nach einer Erweiterung seines Anteils auf 39\% Hauptaktionär der EnBW.

34 Nordostschweizerische Kraftwerke, Énergie Ouest Suisse, Bernische Kraftwerke.

35 Carole Waldvogel, Imposer „l'environnement“. Le travail révélateur des associations alsaciennes (1965-2005), Strasbourg, Presses universitaires de Strasbourg, 2011. 
Modellen, zwischen denen auch kulturelle Hybridisierungen möglich sind, die konkrete Formen annehmen können. Ein erstes grenzüberschreitendes „Bürgerstrom“-Projekt wurde im März 2015 in Colmar umgesetzt. Die Vereine Énergies partagées en Alsace ${ }^{(36)}$ und Fesa Energie ${ }^{(37)}$ haben sich zusammengeschlossen, um eine Energiegenossenschaft zu steuern, die ca. $2600 \mathrm{~m}^{2}$ bürgerfinanzierter Solarzellen verwaltet.

Bei genauerem Hinsehen stellt man fest, dass die Fördermaßnahmen zur Energiewende im Elsass die lokalen Ressourcen unterschiedlich miteinbeziehen. Holzenergie und Wasserkraft wurden gegenüber anderen Quellen wie z. B. Windkraft oder Photovoltaik bevorzugt. So entstand ein erhebliches Ungleichgewicht: 2010 stammten über 90\% der regional produzierten erneuerbaren Energien aus der Wasserkraft (69,5\%) und aus Holzenergie (22\%) ${ }^{(38)}$. Doch der Zweig der Holzenergie ist heute ausgereizt, es sei denn, man geht forstwirtschaftliche Risiken ein (indem man beispielsweise einige spezialisierte Forste allein nach Marktkriterien bewirtschaftet, mit möglichst ertragreichen Baumarten). Die Herausforderung liegt heute darin, einen Energiemix zu schaffen, indem man die anderen Zweige fördert. Die Entwicklung der Windkraft gewinnt dadurch an Bedeutung, besonders angesichts des Kontextes und der Ziele, welche von dem aus den Gesetzen Grenelle 1 und $2^{(39)}$ entsprungenen „Regionalplan für Klima, Luft und Energie" (Schéma régional du climat, de l'air et de l'énergie) vorgegeben wurden. Dieser Plan sieht im Elsass vor, dass bis 2020 26\% des gesamten Energiebedarfs aus „grünen“ Quellen stammen. Zwischen 2009 und 2020 soll laut diesem Plan die Windkraft von 0 auf 22 TOE (Tonnen Öl-Äquivalent) ausgebaut werden, was der Errichtung von ca. fünfzig Windrädern entspricht.

\section{Der Kontext der untersuchten Gemeinde und das Bürgerbeteiligungsmodell}

Die Windkraftausbauzone, auf Französisch zone de développement de l'éolien terrestre $(\mathrm{ZDET})^{(40)}$ getauft, wurde 2008 beantragt und betrifft sechs Gemeinden der Vogesen und, im Elsass, des Département du Bas-Rhin. In der Gemeinde S. werden zehn Windräder mit einer Leistung von jeweils 2 Megawatt errichtet. 2009 leben in diesem Mittelgebirgsdorf 880 Einwohner. Der Sozioökonomische Status ist niedrig. Die Arbeitslosenquote der 17- bis 64-Jährigen liegt bei 17,9\% gegen $10 \%$ im restlichen Elsass. Lediglich 2,7\% der über 15-Jährigen besitzen einen höheren Bildungsabschluss, 29,5\% besitzen keinerlei Bildungsabschluss und 34\% haben eine abgeschlossene französische Berufsausbildung (CAP oder Contrat d'apprentissage professionnel bzw. BEP oder Brevet d'apprentissage professionnel). Es ist bekannt, dass Windkraftanlagen oft in relativ abgelegenen und wirtschaftlich schwachen ländlichen Regionen errichtet

36 Betreibt den Ausbau von Photovoltaikanlagen auf öffentlichen und privaten Gebäuden. Finanziert wird dies über finanzielle Bürgerbeteiligung: http://www.energies-partagees-alsace.coop/ Stand 5. Mai 2015.

37 Deutscher Verein mit Sitz in Freiburg zur Förderung einer dezentralen und demokratischen Energieversorgung auf Basis erneuerbarer Energien: http://www.fesa-geno.eu/ Stand 5. Mai 2015.

38 Guillaume Christen, Cécile Frank, Isabelle Hajek, Philippe Hamman, Mathias Jehling, Maurice Wintz, „Quels enjeux à l'introduction des énergies renouvelables? Une analyse comparative Alsace/ Rhénanie-Palatinat", Revue d'Allemagne et des Pays de langue allemande, 45/1 (2013), S. 81-106.

39 Die Grenelle-Umweltschutzgesetze wurden 2009 und 2010 verabschiedet. Sie enthalten Verpflichtungen und Maßnahmen zu den großen Richtlinien der französischen Umweltpolitik.

40 Instrument der Lokalpolitik um die Errichtung von Windparks zu erleichtern. Wurde 2013 von dem „schéma régional éolien“ abgelöst. 
werden $^{(41)}$. Dies geschieht sicherlich nicht nur aus Gründen der Raumordnung, sondern kann auch als Bestätigung für die Ungleichbehandlung sozial schwächerer Regionen interpretiert werden: Manche sozial schwächeren Gebiete sind entweder empfänglicher für den Bau solcher Anlagen oder sind zumindest weniger imstande, einen Widerstand zu organisieren - ein wichtiger Faktor angesichts der hohen Klagerate gegen Windkraftprojekte in Frankreich ${ }^{(42)}$.

Zwei der zehn Windräder werden über ein Bürgerbeteiligungsmodell von Énergie partagée finanziert, einem Verein, der sich laut eigener Aussage ${ }^{(43)}$ auf „Bürgerinvestitionsmodelle“ bei Windkraftanlagen spezialisiert hat. Die 2012 erfolgten administrativen Genehmigungen und gesetzlichen Freigaben sowie der Rückzug einer Windkraftgegnerbewegung Anfang 2014 machen es nunmehr möglich, Investitionsanteile zu kaufen. Der Bau soll 2015 erfolgen. Die Finanzierung der beiden Windräder wird von einem gemischtwirtschaftlichen Unternehmen gesteuert; diese Finanzierung beruht auf einem dualen Organisationsmodell: Zum einen übernimmt die Gemeinde 40\% der Anteile, mit dem Argument einer Lokalisierung der Energieproduktion - ein Argument, das angesichts der Tatsache, dass die gewonnene Energie in das globale Stromnetz eingespeist wird, nur symbolischen Wert hat - und zum anderen haben die Bürger die Gelegenheit, bis zu 60\% der Anteile selbst zu kaufen. Dieser partizipative Ansatz ist außerdem mit einem regionalen Entwicklungsplan sowie einem Projekt zur Förderung des Tourismus gekoppelt, was dem Windkraftprojekt eine weitere kollektive und gemeinnützige Dimension verleiht. Dafür erhält die Gemeinde eine Auszeichnung, das Ruban du développement durable (Band der nachhaltigen Entwicklung), mit der sie werben kann ${ }^{(44)}$.

Eine klassische Charakteristik der Windkraft in Frankreich ist dessen Zugehörigkeit zu einem spezialisierten industriellen System, welches die Bürger von der Möglichkeit einer Teilnahme an der Energiewende ausschließt ${ }^{(45)}$. Das Modell einer finanziellen „Bürgerbeteiligung“, wie sie von dem Trägerverein zur Begründung seines Kollektiven Ansatzes bezeichnet wird, kann folglich als Vermittlung gesehen werden, um die Akteure für die Problematiken des Umweltschutzes zu sensibilisieren. Dieses Modell erhebt den Anspruch, die Problematik der Klimaerwärmung sowie die möglichen Lösungen in die Reichweite der Bürger zu bringen, setzt dabei aber ein Ausschlusskriterium ein, das wirtschaftlicher und fördertechnischer Natur ist.

\section{Die Untersuchungsmethodik}

Basis der Untersuchung bildet eine Feldstudie ${ }^{(46)}$. Zunächst wurden Interviews mit den Projektträgern durchgeführt, die das finanzielle Bürgerbeteiligungsmodell ent-

41 Siehe Martin J. Pasqualetti, „Morality, Space and the Power of Wind Energy Landscape“, Geographical Review, 90/3 (2000), S. 381-394.

42 Karine Grijol, La faiblesse du vent: impacts, enjeux et contradictions de l'éolien en France, Paris, François Bourin, 2012, S. 95-97.

43 http://energie-partagee.org/le-mouvement/notre-action/ Stand 5. Mai 2015.

44 http://www.rubansdudeveloppementdurable.com/ Stand 5. Mai 2015.

45 Christen/ Hamman, Transition énergétique et inégalités environnementales (Anm. 13).

46 Diese Feldstudie wäre ohne die Mitwirkung von Guillaume Christen, Postdoktorand in Soziologie, und die Unterstützung von Amandine Léonate, die wir an dieser Stelle danken möchten, nicht denkbar gewesen. 
worfen haben, zuallererst mit dem Bürgermeister von S. und seinem Stellvertreter, der mit dem Windkraftdossier befasst ist. Darüber hinaus haben wir Verantwortliche von fünf spezialisierten, lokalen und nationalen Vereinen getroffen, die an diesem Projekt mitarbeiten, um auch die Netzwerke, die die bürgernahen Windkraftkooperationsmodelle steuern, in unsere Untersuchung miteinzubeziehen. Um die Rezeption und die Aneignung dieses Modells zu analysieren, haben wir eine aus 30 Gesprächen bestehende Interviewreihe mit den Einwohnern der Gemeinde S. (12 Frauen und 18 Männer) von Mai bis August 2013 durchgeführt.

\section{Die Problemstellung: Den Einzug der erneuerbaren Energien in die Gesellschaft verstehen}

\section{Die Energiewende als Dispositiv}

Wie die bisherige Forschung gezeigt hat ${ }^{(47)}$, besteht die Besonderheit eines Instrumentes darin, dass es auf einem Netzwerk von Akteuren und Handlungsrepertoires beruht, die zu seiner Entstehung beigetragen haben. Die Rolle der Projektträger besteht nicht nur darin, das Dispositiv zu stützen und Verbreitungsnetzwerke zu entwickeln, um so viele Akteure wie möglich hinzugewinnen zu können; ihre frühzeitige Einbindung bei der Gestaltung beeinflusst die Ausrichtungen des Instrumentes: Sie bestimmen so seine Anwendungsformen. Die verbreiteten Inhalte sind nicht neutral, denn sie stützen sich auf die Gesamtheit der Ressourcen und Bestimmungen, die im Rahmen unterschiedlicher sozialer Kontexte angeeignet wurden. Es ist also möglich, die technischen Entscheidungen der finanziellen Bürgerbeteiligung auf soziale Determinismen wie dem Werdegang der Akteure, ihre Ausbildung, ihre sozialen Beziehungen und ihre Verankerung in verschiedenen Strukturen zurückzuführen. Gleichzeitig bildet das Dispositiv einen Rahmen, der es den Akteuren ermöglicht, sich das Instrument ihrer Praxis entsprechend anzueignen. Die Herausforderung ist, zu erfahren, ob die Akteure die Möglichkeit haben, diesen Rahmen zu verlassen und das Wissen und die Handlungsrepertoires stärker mitzubestimmen.

\section{Ein neuer Ansatz beim Thema ökologische Ungleichheiten}

Die ersten Forschungsarbeiten, welche die Beziehung zwischen sozialen Ungleichheiten und Umwelt problematisieren, stammen aus den Vereinigten Staaten. Sie befassen sich vor allem mit der „Umweltgerechtigkeit“, bei der die Faktoren Umwelt und Natur als Grundsteine für eine sozial gerechte Ordnung verstanden werden ${ }^{(48)}$. Diese Herangehensweise baut hauptsächlich auf der Arbeit von Robert Bullard ${ }^{(49)}$ auf, die zeigt, wie die sozial benachteiligten Bevölkerungsschichten den Umweltrisiken am Stärksten ausgesetzt sind (Bodenverschmutzung, Wohngebiete in der Nähe

47 Pierre Lascoumes, Patrick Le Galès, „From the Nature of Instruments to the Sociology of Public Policy Instrumentation“, Governance, 20/1 (2007), S. 1-21; Matthias WIESER, Das Netzwerk von Bruno Latour: Die Akteur-Netzwerk-Theorie zwischen Science und Technology Studies und poststrukturalistischer Soziologie, Bielefeld, Transcript Verlag, 2012.

48 David Schlosberg, „Theorising Environmental Justice: the Expanding Sphere of a Discourse“, Environmental Politics, 22/1 (2013), S. 37-55.

49 Robert Bullard, Dumping in Dixie: Race, Class, and Environmental Quality (1990), Boulder, CO, Westview Press, 2000. 
von Industrieanlagen, usw.) und dabei zugleich am wenigsten von den Ressourcen profitieren. Diese Definition vernachlässigt jedoch einen weiteren, wichtigen Faktor: Die Beziehungen zwischen den Einwohnern einer bestimmten Region und den Instrumenten, mit denen ebendiese Region nachhaltig weiterentwickelt werden soll und insbesondere Teil der Energiewende werden soll.

Das Aufkeimen der Umweltfrage in der Politik hat einen semantischen Wandel eingeleitet ${ }^{(50)}$, in dessen Zuge die „Ökologie“ zum Symbol für Aktivismus und Bürgerbewegung geworden ist. In diesem Sinne hat Jacques Theys zwischen einer „statischen“ und einer „dynamischen“ Sichtweise der Umwelt unterschieden, um die unterschiedlichen umweltbezogenen Ungleichheiten zu identifizieren ${ }^{(51)}$. Die erste beschreibt den Belastungsgrad durch Umweltrisiken (in der erwähnten amerikanischen Tradition) und die zweite vor allem die Möglichkeit für die Bevölkerung, an der Umweltpolitik aktiv mitzuwirken. Dies erinnert an die Sichtweise von Marianne Chaumel und Stéphane La Branche, für die das Konzept der ökologischen Ungleichheiten vor allem mit den umweltbezogenen sozialen Ungleichheiten zu tun hat ${ }^{(52)}$ : Diese spiegeln sich in den ungleichen Möglichkeiten der verschiedenen Akteure wieder, auf ihre Umwelt Einfluss zu nehmen oder bei Dispositiven zur Realisierung von Umweltprojekten mitzuwirken.

Wir untersuchen so das Potenzial eines Dispositivs - zwei Windräder mit finanzieller Bürgerbeteiligung - die unterschiedlichen Akteure an der Energiewende zu beteiligen, indem wir sowohl die Profile derjenigen untersuchen, die daran mitwirken, als auch derjenigen, die es nicht tun. In welchem Maße dem Instrument Interesse entgegengebracht wird, verdeutlicht die Ungleichheiten beim Verständnis und der Aneignung. Dies führt dazu, dass ein Instrument, welches zunächst inkludierend und kooperativ hätte sein sollen, in Wirklichkeit diskriminierend ist und manche Akteure ausschließt. Wir beleuchten so die Problematik der umweltbezogenen Partizipation unter dem Blickwinkel der ungleichen Beteiligung an der Erzeugung „grüner Energie“. Die Dispositive zur erneuerbaren Energie entsprechen a priori dem Ideal einer nachhaltigen Alternative umso mehr, als sie die Bürger und die Region miteinzubeziehen versuchen. Dennoch können sie neue Ungleichheiten verursachen, indem sie einen Teil der Akteure aus der gemeinsamen Gestaltung eines Gemeinguts ausschließen, welches ein essentielles Element einer geteilten Ökowende-Politik darstellt ${ }^{(53)}$.

In diesem Sinne möchten wir die sozialen Ungleichheiten gegenüber der Instrumente beleuchten, welche die Energiewende ermöglichen sollen, anhand eines Beispiels, dass umso aussagekräftiger ist, als es sich dabei um ein Dispositiv handelt, das

50 Gérard Bellan, Denise Bellan-Santini, Jean-Claude Dauvin, „À propos de quelques utilisations des termes ,inégalités écologiques': simples impropriétés de langage ou accaparement abusif?“, Développement durable et territoires, 9 (2007), URL: http://developpementdurable.revues.org/3426.

51 Jacques Theys, „Pourquoi les préoccupations sociales et environnementales s’ignorent-elles mutuellement. Essai d'interprétation à partir du thème des inégalités écologiques“, in: Pierre Cornut, Tom BAUler, Edwin ZaCCAI (Hg.), Environnement et inégalités sociales, Bruxelles, Université de Bruxelles, 2007, S. 24-35.

52 Marianne Chaumel, Stéphane La Branche, „Inégalités écologiques: vers quelle définition?“, Espace Populations Sociétés, 1 (2008), S. 101-110.

53 Gavin Brown, Peter Kraftl, Jenny Pickerill, Caroline Upton, „Holding the Future Together: towards a Theorisation of the Spaces and Times of Transition“, Environment and Planning A, 44/7 (2012), S. 1607-1623. 
Anreize schaffen soll, sich dabei aber auf Wissensformen und Know-how stützt, mit denen die Akteure in situ nicht unbedingt vertraut sind. Hier wird ein offensichtliches Paradoxon deutlich: Der Bezug zur finanziellen Bürgerbeteiligung kann bestehende soziale Ungleichheiten verstärken oder kumulieren. Dabei haben sich in der Praxis Ungleichheiten bei der Partizipation oder bei der Motivation offenbart, oder unterschiedliche Fähigkeiten, die Relevanz von Umweltfragen zu begreifen.

\section{Die fortschreitende Spezialisierung der finanziellen „Bürgerbeteiligung“}

Bevor wir die sozialen Beziehungen der Akteure zu dem Instrument beleuchten, ist es unverzichtbar, zunächst auf dessen Konzeption und auf die erforderlichen Wissensarten einzugehen, das heißt, das Netzwerk der Akteure zu identifizieren, und insbesondere das der Projektträger. Die Interviews, die mit ihnen geführt wurden, zeigen die zunehmende Bedeutung von Fachwissen, also einer Spezialisierung als Voraussetzung zur Erarbeitung des partizipativen Instrumentes. Das als finanzielle „Bürgerbeteiligung“ bezeichnete Modell beruht vor allem auf technischem, wissenschaftlichem und wirtschaftlichem Spezialwissen, welches die Akteure sich in ihren verschiedenen Sozialsphären aneignen konnten. Wir können nach Héloïse $\mathrm{Nez}^{(54)}$ eine Typologie erstellen, welche zwischen diffusem Fachwissen, kollektivem Wissen und Aktivistenwissen unterscheidet.

\section{Das diffuse Fachwissen}

Es handelt sich um technische Kompetenzen, welche sich die Akteure in ihrem Berufsalltag aneignen. „Diffus“ werden sie genannt, weil sie nicht im Berufsalltag, sondern ausschließlich im Rahmen eines Bürgerprojektes oder Vereinsprojektes Anwendung finden. Dieses Fachwissen wird von einem sozialen Raum in den nächsten übertragen, der sie anerkennen kann. Es handelt sich dabei nicht nur um kognitive Ressourcen, sondern auch um praktisches Know-how. Das Engagement des für das Windkraftprojekt zuständigen stellvertretenden Bürgermeisters der Gemeinde S. ist hierfür symptomatisch: Dieser verrentete ehemalige EDF-Beamte reaktiviert das gesamte technische Wissen und die sozialen Netzwerke, die er während seiner beruflichen Laufbahn aufbauen konnte, um als Selbständiger für die Filiale EDF Énergies Nouvelles als „Projektbeauftragter und Ansprechpartner“ für das Windkraftprojekt zu arbeiten. Dank seines beruflichen Werdegangs als EDF-Agenturleiter in verschiedenen Bezirken im Osten Frankreichs (Longwy, Mülhausen und Schlettstadt) besitzt er ausgeprägte technische Kompetenzen auf dem Gebiet der Energiewirtschaft und verfügt über ein ausgedehntes Netzwerk, das es ihm ermöglicht, ein solches Projekt zu beherrschen und zu leiten. Seine Wahl als Projektleiter wird wie folgt begründet:

„Tja, es wurden Partner gesucht, und da ich Vorkenntnisse auf dem Gebiet habe, habe ich mich gemeldet. Da ich früher für EDF gearbeitet habe, haben sie mich mit EDF Énergies Nouvelles in Verbindung gesetzt. Aber ich hatte früher auch viel mit anderen Unternehmen zu tun. [...] Es gab damals ein anderes Unternehmen, aber [...] die bauen nur kleine Windränder mit 3 Kilowatt, das ist nicht dasselbe. [...] Außerdem war auch C.B. von Électricité

54 Hélö̈se NEz, „Nature et légitimité des savoirs citoyens dans l'urbanisme participatif. Une enquête ethnographique à Paris“, Sociologie, 4/2 (2011), S. 387-404. 
de Strasbourg (ein Stromversorger) mit dabei, den ich sehr gut kannte, so konnten die Kontakte auch sehr viel schneller zustande kommen" (stellvertretender Bürgermeister von S., Mai 2013).

Das angesammelte Wissen leitet unseren Ansprechpartner, ebenso seine Beziehungen zu den lokalen Versorgern, die es ihm ermöglichen, von deren Erfahrungen zu profitieren. Dafür pflegt er seine Kontakte zur lokalen Energiewirtschaft: „Ich hatte erst letzten Dienstag eine Besprechung mit den Vertretern von EDF [...], und am Nachmittag eine mit den Gemeinden der Haute Vallée de la Bruche." Jenseits seiner vormaligen Verantwortlichkeiten als Agenturleiter verfügt er auch über gute Kenntnisse der Bedingungen vor Ort, da er seine Karriere zunächst als Monteur und anschließend als technischer Prüfer begonnen hat. Er verfügt daher über weitreichende Fachkenntnisse des Stromnetzes. Hinzu kommt eine Nähe zu der betroffenen Region, da er bei EDF der „bevorzugte Ansprechpartner der Gemeinden der Haute Bruche“ war, einem Gebiet, zu dem auch die Gemeinde S. gehört. „Ich war für ca. hundert Gemeinden verantwortlich, und dort war es auch, wo wir damals das Windkraftprojekt zusammen mit X [dem Bürgermeister von S.] angestoßen haben. Das war 2003“ (ebd.). An diesem Beispiel wird deutlich, wie die im Laufe des Berufslebens angesammelten Kompetenzen konkret Eingang in das Projekt einer Energiegenossenschaft finden.

Die Mobilisierung kollektiven Wissens: Die Zusammenführung von Fachkenntnissen

Die in Vereinen organisierten Akteure erarbeiten kollektives Wissen, das nicht nur aus der Zusammenführung der individuellen Kenntnisse stammt, sondern auch aus dem Teilen von Projekterfahrungen. Im vorliegenden Fall wurden die operativen Aspekte des Bürgerbeteiligungsprojektes einem Vereinsnetzwerk übertragen, das über ein kollektives Fachwissen auf dem Gebiet verfügt. Diese Übertragung verrät den Status der Naturschutzorganisationen, die zunehmend als Expertenorganisationen fungieren, besonders im Elsass ${ }^{(55)}$. Die Vereine zur Förderung der „Bürgerenergie“, welche mit dem Projekt von S. befasst sind, fokussieren sich nicht auf ein lokales Objekt. Sie erklären, die Energiewende als Ganzes vorantreiben zu wollen. Ihre Funktionsweise ist die eines föderativen Netzwerkes, in dem die großen regionalen oder nationalen Organisationen die Rolle der „harten Kerne“ bei den lokalen Initiativen spielen, und somit die Strukturierung des Wissens und der Kompetenzen erlauben. Im Elsass ist das vor allem Énergies renouvelables citoyennes et solidaires (Ercisol), welche die Bewegung der erneuerbaren Energiegenossenschaften fördert, in dem sie den Anwohnern vorschlägt, zu Teilhabern von Kleinwasserkraftwerken, Photovoltaikanlagen oder Windkraftparks zu werden. Ercisol verfügt inzwischen über mehr als 80 Partner, welche die Gesellschaft ${ }^{(56)}$ mit ihren 650 Beteiligungen mitfinanzieren. Ebenso wie Alsace Énergie Partagée ${ }^{(57)}$ stellt dieses Netzwerk die lokalen Vertretungen von Énergie partagée, einem föderativen Verein, der in Frankreich über fünfzig Einrichtungen zur Förderung von erneuerbaren Energien besitzt.

56 http://www.ercisol.com/ Stand 5. Mai 2015.

57 http://www.energies-partagees-alsace.coop/ Stand 5. Mai 2015. 
Énergie Partagée stellt den lokalen Akteuren die nötigen Werkzeuge zur juristischen Organisation, zur Steuerung und zur wirtschaftlichen Analyse des Bürgerbeteiligungsprojektes sowie für die territoriale Abstimmung zur Verfügung. Das kollektive Wissen, das teilweise extern vorliegt, stützt die regionalen Projekte, wie der Vorsitzende von Ercisol erklärt: „Wir arbeiten an vielen Projekten, sowohl in der Windkraft, der Wasserkraft, als auch in der Photovoltaik. Was S. betrifft, muss man auch sagen, dass wir Teil des Netzwerkes Énergie Partagée sind. [...] Auf nationaler Ebene wissen wir, bei wem wir anklopfen müssen, um an die Informationen zu kommen“ (Mai 2013). Es kann also eine Technisierung der Aktionen der Naturschutzorganisationen festgestellt werden, die sich aus technischem Know-how und Social Engineering zusammensetzt und Erfahrungsaustausch und berufliches Fachwissen der Mitglieder zusammenführt. Letztere bereichern das Netzwerk um ihre Kenntnisse zum Thema Energie: „Unter [den Mitgliedern] gibt es einige Kommunalpolitiker, zum Beispiel der Vize der Verwaltungsgemeinschaft von Montbéliard. Auch haben wir Leute, die beruflich mit Wasserkraft zu tun hatten... Ich habe auch einige Unternehmenschefs..." Der Verein ist somit in der Lage, eine Vorstudie durchzuführen oder seine Rolle als Bauherr vollends wahrzunehmen: „Wir können intern, unter uns, eine Vorstudie machen, um zu sehen, ob ein Vorhaben rentabel ist. Wie haben studierte Ingenieure, was es uns ermöglicht, zu prüfen, ob sich etwas lohnt oder nicht. Wir haben auch ein eigenes, auf Energiethemen spezialisiertes Planungsbüro“ (ebd.).

\section{Das Aktivistenwissen}

Die Akteure können auch auf Aktivistenwissen zurückgreifen, welches im Rahmen von Vereinen oder politischen Verbänden erlernt wurde, und das sie in die Entwicklung des Projektes investieren können, beispielsweise für verwaltungstechnische Themen, die Moderation von Besprechungen, usw. Der Bürgermeister der Gemeinde S., der in politischen Netzwerken aktiv ist (er ist Mitglied der grünen Partei Frankreichs), stützt sich so auf ein sektorenübergreifendes Verständnis der Energiewende, indem er sie in ein Projekt zur nachhaltigen Entwicklung der Region einbindet: „Wir tun es für den fairen Handel, weil das mein Steckenpferd ist, und ich habe mich um die Bewerbung gekümmert. [...] Ich werde jetzt wieder die Bewerbung für das ruban du développement durable zusammenstellen, das uns 2006 bereits verliehen wurde“ (Bürgermeister der Gemeinde S., März 2013). Die praktische Effizienz des Aktivistenwissens besteht in seiner Anwendbarkeit auf andere Domänen. Hier wird es benutzt, um die Windkraft in einem regionalen Entwicklungsprojekt zu platzieren, um ihm die doppelt legitimierende Dimension des lokalen und zugleich globalen Nutzens $\mathrm{zu}$ verleihen. Dementsprechend wird das Aktivistenwissen auch als Fachwissen bei der Bürgerpartizipation genutzt. So nimmt der Bürgermeister zum Beispiel an dem Kollektiv Cléo teil, dem club des collectivités locales éoliennes françaises (Verein der französischen Windkraftgemeinden), gegründet 2003, der die Erfahrungen in Sachen Bürgerwindkraft bündelt und den Kommunalpolitikern ingenieurtechnisches Wissen und Konsultationsmethoden bietet.

Das von den Trägern des Windkraftprojektes genutzte Wissen verbindet also juristische, technische und kollektive Kompetenzen sowie Aktivistenwissen. Dies kennzeichnet die für die Teilnahme an einem solchen Projekt zunehmende Notwendigkeit der Spezialisierung, bei der das partizipative Instrument von einem technischen 
Repertoire geprägt ist. Dementsprechend muss man die Verbreitung und Rezeption dieses Wissens durch die Bürger, die Umstände seiner Demokratisierung bedenken; mit anderen Worten, in welcher Beziehung stehen die Bewohner von S. zu diesen Kompetenzen, die dank beruflicher Register entstanden sind, die ihnen fremd sind?

\section{Unterschiedliche Aneignungsformen, ein Indikator für ökologische Ungleichheiten}

Derek Bell et al. haben auf eine doppelte Diskrepanz verwiesen, um die lokalen Protestbewegungen gegen Windkraft zu erklären ${ }^{(58)}$ : eine soziale Diskrepanz, durch den Unterschied zwischen prinzipieller Unterstützung der erneuerbaren Energien in den Umfragen in abstracto und der wenig positiven Wahrnehmung der Windparks in concreto; und eine individuelle Diskrepanz, durch den Unterschied zwischen der Unterstützung der Windkraft im Allgemeinen und einer Ablehnung von lokalen Bauprojekten, die oft als Verschandelung der Landschaft wahrgenommen werden ${ }^{(59)}$ und denen vorgeworfen wird, die Umwelt und die Gesundheit der Anwohner zu belasten (Geräuschentwicklung, elektromagnetische Wellen, Licht-Schatten-Wirkung durch die sich drehenden Rotoren). Der NIMBY-Effekt (not in my backyard - nicht in meinem Garten) kann also nicht die einzige Erklärung für die soziale Diskrepanz sein ${ }^{(60)}$ - Erklären lässt sich diese zum einen auch durch das „demokratische Defizit“, und zum anderen durch die Tatsache, dass die Unterstützer der Windkraft „qualifizierte Unterstützer“ bzw. Kenner der Windkraft sind, so dass das Thema letztlich einem technisch informierten Publikum vorbehalten bleibt.

Aus dem Blickwinkel der Einwohner bleibt die Bürgerbeteiligung bei dem Windkraftprojekt in S. folglich dem Spezialistennetzwerk vorbehalten, der es angestoßen hat. Verdeutlicht wird dies durch die starken Ungleichheiten bei dem Verständnis des Dispositivs. Diese Grenzen verraten, wie wichtig die Integration der Bürger ist, egal ob über eine finanzielle Beteiligung oder über die Mitarbeit am Projekt, um ein solches Unterfangen im Bereich der erneuerbaren Energien durchzuführen. Sie ist der Schlüssel zur sozialen Akzeptanz lokaler Initiativen und, weiter gefasst, zu einer stärkeren und konkreteren Bewusstwerdung der anstehenden energetischen Herausforderungen. Gegenüber technischen Legitimationsformen, deren Verständnis nicht selbstverständlich ist, steht die Verbundenheit zu einer Region und dem, was das soziotechnische System konkret fassbar macht (visuell, wirtschaftlich...) ${ }^{(61)}$.

\section{Die Legitimität zu handeln und teilzunehmen ist nicht selbstverständlich}

Die Bürgerpartizipation an einem Energieprojekt ist keine Selbstverständlichkeit: Dies äußert sich durch ein Gefühl der Illegitimität oder des Desinteresses, das von

58 Derek Bell, Tim Gray, Claire Haggett, Joanne Swaffield, „Re-visiting the ,Social Gap؛: Public Opinion and Relations of Power in the Local Politics of Wind Energy“, Environmental Politics, 22/1 (2013), S. 115-135.

59 Bernd MöLler, „Changing Wind Power Landscapes. Regional Assessment of Visual Impact on Land Use and Population in Northern Jutland“, Applied Energy, 83/5 (2006), S. 477-494.

60 Patrick Devine-Wright, „Beyond NiMBYism: Towards an Integrated Framework for Understanding Public Perceptions of Wind Energy“, Wind Energy, 8/2 (2005), S. 125-139.

61 Alexandra M. Gormally, Colin G. Pooley, Duncan Whyatt, Roger Timmis, „Attitudes Towards Community Renewable Energy in Cumbria“, Local Environment, 19/8 (2014), S. 915-932. 
einigen Einwohnern zum Ausdruck gebracht wird. Es stellt sich folglich die Frage nach der Berücksichtigung der Gebrauchserfahrungswerte der Einwohner beim Errichtungsprozess der Anlagen zur Erzeugung erneuerbarer Energie in einem bestimmten Landabschnitt oder einer Gemeinde ${ }^{(62)}$, um ihre Verbreitung und konkrete Aneignung auch jenseits ihrer Initiatoren und Techniker zu erleichtern, bzw. jenseits der Aktivisten oder Vereine, die sie bekämpfen oder ihre eigenen, alternativen soziotechnischen Werkzeuge stellen ${ }^{(63)}$.

Zum einen generiert die Delegierung des Dispositivs an einen nicht-lokalen Expertenverband eine erste soziale Distanz. Wie Anthony Giddens erklärte, beruht die Besonderheit eines Expertensystems auf einer räumlichen und zeitlichen Distanz ${ }^{(64)}$. Das Delegieren an Kompetenznetzwerke löst die sozialen Beziehungen aus ihrem lokalen und zeitlichen Kontext. In unserem Fall ist die Umsetzung des partizipativen Instrumentes nicht mehr an einen den Einwohnern bekannten Ort geknüpft: Sie wird stattdessen einem Netzwerk professioneller und nicht-lokaler Akteure delegiert. Auch die Zeichnung der Anteile weist diese Distanz auf, insofern als sie per Internet geschieht. Einzige verbleibende Identifikationspunkte der Bevölkerung sind somit allenfalls der Bürgermeister oder die Gemeinde: „Das Dorf, der Bürgermeister kümmert sich darum. Sie werden die beiden Windräder kaufen, das wird sich positiv auswirken, das ist gut für das Dorf“ (Einwohner von S., Juli 2013). Aus der Perspektive der Einwohner ist der Bürgermeister der einzige sichtbare und identifizierbare „Verbindungspunkt“ des soziotechnischen Kollektivs. Durch diese Verbindungspunkte Kommuniziert das Expertensystem, um mit den „normalen“ Akteuren, den Bürgern, ein Vertrauensverhältnis aufzubauen, indem er ihnen die Möglichkeit gibt, die Expertenmeinungen der Spezialisten im Hinblick auf die lokalen Verhältnisse zu überprüfen ${ }^{(65)}$. Hier bleibt das für die finanzielle Bürgerbeteiligung verantwortliche Kompetenzennetzwerk der Kollektive Ercisol und Alsace Énergie Partagée unsichtbar.

Die Probleme der Einwohner bei der Identifizierung des Kollektivs, das die Bürgerbeteiligung organisiert, sind für die herrschende soziale Distanz bezeichnend: „Wenn wir da mitspielen, wen muss man da fragen? Wer organisiert das? Das muss ja die Gemeinde sein; ich denke, man wird wohl beim Rathaus nachfragen müssen, um Anteile zu kriegen. Die steuern doch alles?..." (Einwohner von S., Juli 2013). Diese Ratlosigkeit verrät nicht nur die mangelnde und ungenaue Kenntnis des Instrumentes, sondern auch eine Fremdheit gegenüber den zugrundeliegenden Verbänden. Dieser „Laienstatus“ in der Beziehung zum Instrument kontrastiert stark mit dem der Akteure der Vereine, die, ganz im Gegensatz zu den Einwohnern, eine große Selbstverständlichkeit und Vertrautheit mit dem Instrument aufweisen: „Hören Sie, wir haben einen Internetauftritt, die Leute können das Teilhaberformular herunterladen und uns zuschicken, und wir entscheiden danach im Komitee“ (Vorsitzender von Ercisol, Mai 2013). Hier wird die Diskrepanz zwischen den Gebrauchslogiken, so

62 Carrie BeHAR, „Utilising Resident Feedback to Inform Energy-Saving Interventions at the Barbican“, Local Environment, 19/5 (2014), S. 539-559. 
wie sie sich die Einwohner vorstellen, und denen, die zuvor von den Initiatoren und Organisatoren festgelegt wurden, deutlich.

Manche Einwohner gehen sogar so weit, ihr eigenes Unvermögen, an dem Dispositiv teilzunehmen, hervorzuheben: „Der Bürgermeister weiß, was er tut. Ich kenn mich da nicht aus, kann da nichts groß machen, weiß nicht so recht, bin da nicht an meinem Platz. Das ist nichts für kleine Bürger wie wir, das ist etwas für Spezialisten“ (Einwohner von S., August 2013). Diese Aussage ist aus mehreren Gründen interessant: Schon der erste Satz verweist auf die soziale Stellung und den Symbolischen Status des Bürgermeisters - und die Anerkennung seiner Legitimität ist aus Formulierungen wie „Der Bürgermeister weiß, was er tut“ ersichtlich. Ein weiterer Aspekt ist, dass die Windkraft für Akteure, die nicht mit dem Thema der erneuerbaren Energien vertraut sind, ein technisches Fachwissen vorauszusetzen scheint, das nur „Spezialisten“ vorbehalten ist. Außerdem sind die Einwohner der Gemeinde S. nicht mit alternativen Finanzierungsmodellen wie der finanziellen Bürgerbeteiligung vertraut: „Finanzielle Bürgerbeteiligung? Was ist das? Ich verstehe nicht so ganz, wie das geht..." (Einwohnerin von S., August 2013). Indem sie sich inkompetent fühlen, unterschätzen die Akteure ihr eigenes Potenzial, sie fühlen sich fehl am Platze und glauben, keine legitime Berechtigung zu haben, sich an dem Projekt, so wie es konzipiert wurde, zu beteiligen - folglich bringen sie sich auch nicht ein.

Die Ursachen für die lokalen Protestbewegungen, die häufig bei Windkraftprojekten auftreten, darf man daher nicht ausschließlich in reinen Umweltbewusstseinsregistern suchen, sondern auch in den Interaktionen zwischen kollektiven Identitäten, Ortsverbundenheit und einer unterschiedlichen Wahrnehmung der Region, die in Bezug auf das sozio-ökonomische Profil der verschiedenen anwesenden Akteure und die verfügbaren oder nicht-verfügbaren Ressourcen einen weiteren strukturierenden Faktor darstellen ${ }^{(66)}$.

\section{Know-how und Lebenseinstellung bestimmen über Partizipation und Engagement}

Die Ungleichheiten in der Aneignung lassen sich auch an dem Verständnis ablesen, das die Akteure von der Funktionsweise des Dispositivs haben. Die meisten befragten Einwohner haben nur eine „nominale und partielle Kenntnis“(67), das heißt, dass ihnen die Existenz des Instrumentes bekannt ist, seine genaue Funktionsweise aber nicht. Dies führt zu einer gemeinsamen Vorstellung, nach der der produzierte Strom lokal genutzt werden soll und der öffentlichen Infrastruktur der Gemeinde zugutekommen soll - in Wirklichkeit jedoch soll er in das nationale Stromnetz eingespeist werden: „Das wird auch gut für unser Dorf sein. Da werden wir Geld sparen können, wenn wir unseren eigenen Strom haben. Damit werden wir zum Beispiel das Rathaus oder den Festsaal mit Strom versorgen können“ (Einwohnerin von S., Juni 2013) oder auch: „Die Windräder, die können uns viel bringen, vor allem für die Straßenbeleuchtung und [...] auch für die Dorfbewohner“ (Einwohnerin von S., Juni 2013). Diese Akteure haben keinerlei Erfahrung oder praktisches Wissen, dass ihnen bei dem Verständnis

66 Antje Отто, Markus Leibenath, „The Interrelation between Collective Identities and Place Concepts in Local Wind Energy Conflicts“, Local Environment, 19/6 (2014), S. 660-676.

67 Im Sinne von Pierre Bourdieu, Distinction: A Social Critique of the Judgement of Taste (1979), London, Routledge, 2010. 
der Funktionsweise der Windkraftanlage helfen könnte. Die Möglichkeit, gar Teilhaber zu werden, erscheint ihnen folglich äußerst unwahrscheinlich bzw. vollkommen undenkbar: „Sehen Sie, ich hätte nicht gedacht, das sowas möglich ist, Teilhaber der Windkraftanlage zu werden. Ich wusste, dass das bei Photovoltaikanlagen geht, aber nicht bei Windkraftanlagen (Einwohner von S., August 2013). Die finanzielle Bürgerbeteiligung hängt also von der Kenntnis bzw. Unkenntnis der Trägervereine der sog. „Bürgerinitiative“ ab.

Die Miteinbeziehung in das Dispositiv setzt ein gewisses Know-how und eine gewisse Lebenseinstellung voraus, ohne die ökologische Demokratie undenkbar ist: Es reicht nicht, für oder gegen das Instrument zu sein; man muss die Bereitschaft haben, sich zu engagieren, einem Verein beizutreten und mitzuarbeiten, und dieses Engagement setzt den Einsatz von praktischem Wissen voraus ${ }^{(68)}$. Das Engagement ist nur dann sinnvoll, wenn die Akteure auch über das Know-how verfügen, das es ihnen ermöglicht, die Herausforderungen des sog. „Bürger“-Windkraftprojektes zu begreifen. Manche Einwohner hinterfragen zum Beispiel den Nutzen der Windkraftanlage für die Gemeinde: „Und was bringen uns die Windränder? Und wem? EDF Énergies Nouvelles? Und wir, die Bevölkerung, was bekommen wir?" (Einwohner von S., Juni 2013). Für Letztere ergibt das Projekt nur wenig Sinn; die „aktivistische“ Dimension der finanziellen Bürgerbeteiligung wird hier nicht wahrgenommen.

\section{Die wirtschaftliche Bremse}

Auch die wirtschaftliche Dimension des Instrumentes ist von Bedeutung, da der Ankauf von Anteilen ein finanzielles Engagement voraussetzt. Der Ankaufpreis kann für die Bewohner von S. ein Hindernis darstellen. Der wirtschaftliche Kontext dieser ländlichen Gemeinde ist für die Vogesen symptomatisch: Die Industrie befindet sich in einer Rezession (insbesondere die Textilindustrie, aber auch die Holzindustrie). Das mittlere Nettoeinkommen pro steuerrechtlichem Haushalt liegt laut dem französischen Statistikamt Institut national de la statistique et des études économiques für 2009 bei 16.419 Euro und somit ein Drittel unter dem elsässischen Durchschnitt von 24.352 Euro. Die Entscheidung kann also auch mit dem Ankaufpreis zusammenhängen, der den Einwohnern zum Zeitpunkt der Befragung 2013 nicht bekannt ist: „Ich kaufe gerne einen oder mehrere Anteile, aber das hängt auch vom Preis ab, ich kann mir nicht viele Extras leisten" (Einwohner von S., August 2013). Diese schwierigen wirtschaftlichen Verhältnisse kontrastieren stark mit denen, die der Vorsitzende von Ercisol bei der Beschreibung einer kurzfristigen Finanzierungsaktion schildert: „In den Vogesen mussten wir sehr schnell handeln, weil der Verkäufer aus steuerlichen Gründen vor dem 31. Dezember 2012 unterschreiben wollte. [...] Es blieb also keine Zeit, mit einer Bank darüber zu verhandeln. Ich habe dann bei uns eine kleine Umfrage gestartet [...] und ca. 10 Minuten später hatte ich 400.000 Euro zusammen. Wer hätte das gedacht! Das erstaunt mich noch heute“ (Mai 2013).

Neben dem kollektiven Fachwissen scheint der Verein also auch recht finanzstark zu sein. Im Zuge desselben Interviews äußerte sich der Vorsitzende von Ercisol auch zum Preis der Anteile: „Wir haben den Preis pro Anteil auf 500 Euro festgesetzt, aber wie

68 Cécilia Claeys, Marie Jacqué (Hg.), Environmental Democracy Facing Uncertainty, Brussels, PIE Peter Lang, 2012. 
gesagt: die Leute können eine kaufen, oder zwanzig...“( $(e b d$.$) . Die sozioökonomischen$ Kapazitäten beschränken also die Mitmachmöglichkeiten der Akteure deutlich und schaffen dadurch eine Form von Ausgrenzung, die dem bürgernah gewollten Bürgerbeteiligungsprojekt schadet. Die Ungleichheiten hängen folglich stark mit den Modalitäten und Organisationskriterien des Instrumentes zusammen.

\section{Vom Aktionsrahmen ignoriertes, praktisches Wissen}

Die Anwohner von S. vollziehen eine Art Aneignung durch ihr praktisches Wissen: Die Materialisierung der Windräder und ihre Visualisierung in einem räumlichen und sozialen Kontext ermöglicht es den Nutzern, den Fluss der Materialien, ihre Herstellung und ihre Verteilung zu erfahren und sich anzueignen ${ }^{(69)}$. Der Bau der zwei Windräder veranlasst die Einwohner, sich ein konkretes Bild von der Produktionsstätte zu machen und sich ein Verteilungssystem zwischen Windrädern und öffentlichen Gebäuden vorzustellen: „Ich denke, das bringt der Festhalle was, selbst dem Rathaus, alle Gebäude werden den Strom benutzen können“, erklärt eine Einwohnerin der Gemeine S. (Juni 2013). Diese kognitive Aneignung der Energienetzwerke kann als eine Form von Ressourcen- und Raumbezogenes Wissen interpretiert werden, durch die die Akteure ihre Gemeinde in einen regionalen Metabolismus einordnen, in dem sie ein Verständnis und eine Vorstellung der Ressourcenströme haben.

Diese Wissensformen stehen jedoch im Widerspruch zu den vordefinierten Anwendungsformen der Initiatoren. Wenn die Träger Anwendungsformen und Vorhaben formulieren, legen sie auch eine Hierarchie zwischen den verschiedenen, unterschiedlich gewerteten Wissensformen fest. So wie das Dispositiv gestaltet ist, wird die finanzielle Teilnahme anerkannt, das Wissen um den regionalen Metabolismus aber nicht. Diese Ausgrenzung offenbart bei Umweltprojekten die Existenz eines Machtgefüges und einer Dominanz, welche den Vereinen und Verbänden zuspielt, die zunehmend als Experten auftreten. Das Verhältnis der Nutzer zur Energieproduktion wird hingegen ignoriert. Das ist ein Zeichen dafür, dass die Entwicklung hin zu einem demokratischen Prozess der Einbindung der Bürger, der auch „Laien“ miteinbezieht, nicht selbstverständlich ist ${ }^{(70)}$, und das die Professionalisierung der „alternativen“ Finanzinstrumente sich zunehmend von den Bedürfnissen und Möglichkeiten der Einwohner entkoppelt.

Die Beschränkung des Instruments auf die Träger von Spezialwissen kann den Bereich der partizipativen Energie gegenüber den bildungstechnisch und wirtschaftlich benachteiligten Akteuren weiter verschließen. Wenn die Gestaltung der Instrumente zur Energiewende auch weiterhin ohne Berücksichtigung eines gemeinsamen Wissensregisters gestaltet wird und auf eine Logik von Expertenwissen und Finanzstärke setzt, wird die Möglichkeit einer Aneignung durch ein breiteres Publikum umso unwahrscheinlicher werden. Der Spezialisierungsgrad und die gesellschaftliche Stellung der Träger des vorliegenden Projektes sind ein Hinweis auf die Existenz von Ausgrenzungsmechanismen, die zudem von einer weiteren, wirtschaftlichen Exklusion beim Anteilskauf noch verschärft werden.

69 L. Dobigny, „Changement énergétique et rapport au monde“ (Anm. 12).

70 Michel Callon, Pierre Lascoumes, Yannick Barthe, Acting in an Uncertain World. An Essay on Technical Democracy (2001), Cambridge-London, The MIT Press, 2009. 


\section{Fazit}

Der Fall des Windparks der Gemeinde S. hat gezeigt, dass die Projektträger technischen Vereinsnetzwerken angehören, die außerdem auf kooperative Prozesse spezialisiert sind, was es ihnen ermöglicht, über die nötigen Instrumente und das nötige Fachwissen im Hinblick auf eine Energiewende zu verfügen, die sich via eines Bürgerbeteiligungsmodells als Alternative zu den großen, zentralisierten Konzepten darstellt. Sie greifen dazu auf ein umfangreiches Instrumentarium zurück, welches es ihnen ermöglicht, sich der technischen und soziologischen Aspekte des Projektes zu bemächtigen, indem sie den Windkraftpark als kollektives regionales Projekt inszenieren. Die Professionalisierung der Bezugssysteme entfernt diese Akteure von den Gebrauchslogiken der Einwohner der betroffenen Gemeinde, die nicht Teil dieses Kollektivs sind. Hinter dem Diskurs einer gleich zweifach nachhaltigen Energieerzeugungsalternative (inhaltlich, wegen der nachhaltigen Energieerzeugung, und Verfahrenstechnisch, wegen der Möglichkeit der Bürgerbeteiligung), verbirgt sich hauptsächlich ein Teilhaber-„,Klub“, der aus ressourcenreichen Individuen besteht (sozial, finanziell, netzwerkbezogen, usw.) und dessen regionale Verankerung relativ ist (ein lokaler Windkraftpark, dessen Teilhaber jedoch tendenziell eher aus anderen Regionen kommen).

Das Instrument wird außerhalb der Erlebniswelt und der Praxis der großen Mehrheit der lokalen Akteure entwickelt, was der Debatte um ökologische Ungleichheiten eine neue Dimension verleiht. Die Analyse hat gezeigt, dass die unterschiedlichen Beziehungen der Akteure zur Umwelt strukturell und organisatorisch von der unterschiedlichen Beherrschung der zur Umsetzung eines solchen kooperativen Projektes nötigen Instrumente abhängen. Die Mobilisierung der lokalen Bevölkerung in Sachen kollektiver und kooperativer Energieerzeugung wird durch Modalitäten und Teilnahmebedingungen gebremst, die ein gewisses Fachwissen voraussetzen. So wie sie aktuell gestaltet sind, setzen die Bedingungen zur Teilnahme und zum Engagement das Vorhandensein eines gewissen Know-hows und einer bestimmten Lebenseinstellung voraus, wie sie für die Vereine und Aktivistengruppierungen charakteristisch sind. Die Ungleichheit beruht daher vor allem in der Fähigkeit bzw. Unfähigkeit der Akteure, ihre Beschäftigung mit dem Thema in konkretes Handeln umzuwandeln. Doch weder das Interesse, noch die Fähigkeit, sich zu engagieren, sind selbstverständlich: Hierzu die richtigen „Techniken“ abrufen zu können ist ein besonders entscheidender Faktor. Es geht dann nicht mehr nur um Ungleichheiten bezüglich der Werte und Interessen im Bereich Umwelt und nachhaltige Entwicklung( ${ }^{(71)}$, sondern auch bezüglich der Fähigkeit, sich einzubringen und zugunsten der Energiewende zu wirken. Die Einschränkungen des Instrumentes werfen zudem die Frage der ökologischen Demokratie auf. Der gesteckte Aktionsrahmen schließt die Bevölkerung der betroffenen Gemeinde von der Möglichkeit aus, ihr eigenes Gebrauchswissen zum Thema Energie einzubringen, das heißt ihr Verständnis, nach dem die Windräder zur energetischen Unabhängigkeit der Gemeinde beitragen werden (auch wenn es sich dabei um eine Fehlannahme handelt). Dies begrenzt die Möglichkeiten, das Instrument einem weitgefassten Publikum zu vermitteln.

71 Philippe Hamman, Cécile Frank, Marie Mangold, „Les trajectoires de conversion écologique face aux enjeux socio-économiques du ,logement durable‘ en France“, VertigO, 14/2 (2014), URL: http:// vertigo.revues.org/15018. 
Die Modalität der Partizipation, die größtenteils um ein Finanzinstrument herum gestaltet wurde, stellt die Frage nach dem Primat der wirtschaftlichen Interessen gegenüber der ökologischen. Dieser Zustand, der dem Handlungsrepertoire der nachhaltigen Entwicklung oft vorgeworfen wird ${ }^{(72)}$, wird in der Ökonomisierung der ökologischen Problematiken deutlich, bei der die Partizipation hauptsächlich aus wirtschaftlichen und präskriptiven Hebeln besteht, die einen perfekt informierten homo ecologicus voraussetzen, der stets rational handelt ${ }^{(73)}$. Unsere Untersuchung offenbart im Bereich der Energieerzeugung ganz konkret dieses oft unterschwellige Postulat einer sozialen Rezeption der umwelttechnischen Innovationen, welche sich über die Diversität der sozialen Kontexte hinwegsetzt.

Betrachtet man die Lage aus der Sicht der Einwohner, und nicht aus der Sicht der Projektträger, ist die Partizipation beim untersuchten Projekt äußerst gering, beinahe beschränkt auf die bloße Information, bzw. teilweise gar nicht vorhanden. In diesem Kontext ist das Angebot eines Anteilkaufs lediglich eine Möglichkeit, die Nachteile der Errichtung eines Windparks zu kompensieren - Ein Windpark, der, abgesehen von den zwei bürgerfinanzierten Windrädern mit all ihren bereits erwähnten Einschränkungen, durch die anderen acht Windräder eine industrielle Dimension aufweist. So gesehen besteht die Bürgerbeteiligung aus einem Instrumentarium industrieller Ökologie( ${ }^{(74)}$, dessen Ziel es ist, die „grünen Technologien“ sozial und lokal zu verankern, das heißt, die Auswirkungen der Industrie auf die Umwelt zu begrenzen, ohne dabei jedoch die Grundlagen des bestehenden Wirtschaftssystems infrage zu stellen. Der Übergang vom „Erneuerbaren“ zum „Alternativen“ ist kein Selbstläufer ${ }^{(75)}$, und dies geht weit über die Experten- oder Entscheiderdebatten über Nachhaltigkeitskriterien hinaus ${ }^{(76)}$; die Gestaltung und Anfechtung dieser Realitätswahrnehmungsmuster hängen in der Tat mit Wissensformen zusammen, die nicht von allen geteilt werden. Dies ist auch eine Einladung, die Nachhaltigkeit aus der Perspektive einer gewissen „Enttechnisierung“ zu betrachten ${ }^{(77)}$, und in der Technologie nicht unbedingt „die“ Antwort des menschlichen und sozialen Fortschritts auf die aktuelle Umweltkrise zu sehen.

\section{Danksagung}

Diese Forschungsarbeit wurde mit Unterstützung des Europäischen Fonds für regionale Entwicklung (EFRE) und dem Interreg IV Programm für die Region Oberrhein in Zusammenarbeit mit der Wissenschaftsoffensive der Trinationalen

72 Pearsall/ Pierce/ Krueger, „Whither Rio+20?“(Anm. 2).

73 Jean-Baptiste Cомву, „Faire du bruit sans faire de vagues“, Communication, 31/2 (2013), URL: http:// communication.revues.org/4439.

74 Xuemei BaI, Heinz Schande, „Urban Ecology and Industrial Ecology“, in: Ian Douglas, David Goode, Mike Houck, Rusong Wang (Hg.), The Routledge Handbook of Urban Ecology, London, Routledge, 2015, S. 26-37.

75 Leland Luther Glenna, Robert Roy Thomas, „From Renewable to Alternative: Waste Coal, the Pennsylvania Alternative Energy Portfolio Standard, and Public Legitimacy", Society and Natural Resources, 23/9 (2010), S. 856-871.

76 Tomas Moe Skjølsvold, „What We Disagree about When We Disagree about Sustainability“, Society and Natural Resources, 26/11 (2013), S. 1268-1282.

77 Marina Fischer-Kowalski et al., Gesellschaftlicher Stoffwechsel und Kolonisierung von Natur, Amsterdam, G+B Verlag, 1997. 
Metropolregion Oberrhein (TMO): Projekt C31 Plan-Énergies renouvelables (Plan-EE, Entwicklung eines Planungstools zur Einführung der erneuerbaren Energien in der Region Oberrhein, 2012-2015) durchgeführt. Weiterführende Informationen finden Sie auf der Website: http://www.plan-ee.eu/

\section{Zusammenfassung}

Dieser Artikel beleuchtet die Ungleichheiten in der Partizipation der verschiedenen sozialen Akteure bei der Produktion des Allgemeinguts der "grünen Energien“. Insbesondere stellt er die Frage nach den unterschiedlichen Bezügen der Bewohner regionaler Räume zu den Instrumenten, mit deren Hilfe die Energiewende vollzogen werden soll. Die Analyse einer Fallstudie aus dem ländlichen Raum im Elsass ermöglicht es, den oft vorgeschobenen Dualismus zwischen den zwei großen „Energie-Modellen“, dem zentralisierten Modell und dem dezentralisierten, dem industriellen und dem „bürgernahen“, oder auch dem französischen und dem deutschen, zu nuancieren. Wir untersuchen die Umsetzung eines Instrumentes zur finanziellen Bürgerbeteiligung bei einem „bürgernahen" Windparkprojekt, dessen Ziel es ist, die Bewohner der betroffenen Gemeinde in die Energiewende miteinzubeziehen. In der praktischen Umsetzung scheitert dies an unterschiedlichen Partizipationsgraden, aber auch an der unterschiedlichen Aneignung von Rechtfertigungsregistern, die sich oft ohne das nötige Fachwissen dem Laien nicht erschließen. Diese Formen der Ungleichheit zeigen nicht nur, dass die verschiedenen Akteure von der Schädigung der Umwelt und der damit verbunden Risiken unterschiedlich stark betroffen sind; sie werfen auch die Frage nach den Auswirkungen der umweltbezogenen sozialen Ungleichheiten auf, die von den unterschiedlichen Wissensständen und Vorgehensweisen der verschiedenen Akteure sowie von den ungleichen Partizipationsmöglichkeiten und dem unterschiedlichen Mobilisierungsgrad bei sogenannten „umweltfreundlichen“ Projekten geprägt sind.

\section{Résumé}

Cet article éclaire l'inégale participation des acteurs sociaux à contribuer à la production de l'"énergie verte " comme bien commun. Plus précisément, il questionne le rapport différencié des habitants dans des espaces locaux face aux instruments censés opérer la transition énergétique. S’appuyer sur une étude de cas dans un territoire rural en Alsace permet de nuancer l'opposition binaire souvent avancée entre deux "modèles " énergétiques, centralisé et déconcentré, industriel et "citoyen", ou encore français et allemand. Nous interrogeons la mise en cuvre d'un instrument d'actionnariat populaire autour d'un projet éolien "citoyen", dont l'ambition est d'associer les habitants de la commune concernée à la transition énergétique. Concrètement, ceci se heurte à des inégalités de participation mais aussi d'appropriation des registres de justification, qui demeurent problématisés à partir d'identités professionnelles expertes. Ces formes d'inégalités ne renvoient pas uniquement à une exposition inégale des acteurs face à des nuisances et des risques environnementaux; elles questionnent l'enjeu des inégalités sociales face à l'environnement, c'est-à-dire des rapports de connaissance ou de pratiques différenciés entre acteurs et des capacités inégales de participation et de mobilisation autour de projets environnementaux. 